\title{
THE $\mathrm{SL}_{3}$ COLORED JONES POLYNOMIAL OF THE TREFOIL
}

\author{
STAVROS GAROUFALIDIS, HUGH MORTON, AND THAO VUONG
}

(Communicated by Daniel Ruberman)

\begin{abstract}
Rosso and Jones gave a formula for the colored Jones polynomial of a torus knot, colored by an irreducible representation of a simple Lie algebra. The Rosso-Jones formula involves a plethysm function, unknown in general. We provide an explicit formula for the second plethysm of an arbitrary representation of $\mathfrak{s l}_{3}$, which allows us to give an explicit formula for the colored Jones polynomial of the trefoil and, more generally, for $T(2, n)$ torus knots. We give two independent proofs of our plethysm formula, one of which uses the work of Carini and Remmel. Our formula for the $\mathfrak{s l}_{3}$ colored Jones polynomial of $T(2, n)$ torus knots allows us to verify the Degree Conjecture for those knots, to efficiently determine the $\mathfrak{s l}_{3}$ Witten-Reshetikhin-Turaev invariants of the Poincaré sphere, and to guess a Groebner basis for the recursion ideal of the $\mathfrak{s l}_{3}$ colored Jones polynomial of the trefoil.
\end{abstract}

\section{INTRODUCTION}

The initial goal of this paper is to provide a supply of explicit quantum invariants so as to help in formulating and testing a number of conjectures. The most readily approachable knots in this context are the $(m, n)$ torus knots, particularly when $m=2$. The aim is to give explicit details for the $\mathfrak{s l}_{3}$ invariants, as these are potentially the simplest case after the more readily available colored Jones $\left(\mathfrak{s l}_{2}\right)$ invariants.

There is a general method of Rosso and Jones to determine any quantum invariant of a torus knot. For the invariant of the $(m, n)$ torus knot with quantum group module $V$ their calculations require knowledge of the decomposition of the module $\psi_{m}(V)$ into irreducible representations. This is a combinatorial problem depending on the quantum group and the choice of $V$, which does not always have a readily available explicit formula.

We give here an explicit formula where $m=2$ and $V$ is a general irreducible $\mathfrak{s l}_{3}$ module; from this we are able to give a detailed estimate for the extreme degrees of the resulting Laurent polynomial invariant.

Subsequently the second author reformulated some combinatorial work of Carini and Remmel CR98] describing $\psi_{2}(V)$ for the irreducible $\mathfrak{s l}_{N}$ modules which correspond to partitions with two parts. This recovers the explicit formulae for $\mathfrak{s l}_{3}$ and also allows us to extend them to $\mathfrak{s l}_{N}$.

Received by the editors December 6, 2010 and, in revised form, September 30, 2011.

2010 Mathematics Subject Classification. Primary 57N10; Secondary 57M25.

Key words and phrases. Colored Jones polynomial, knots, trefoil, torus knots, plethysm, rank 2 Lie algebras, Degree Conjecture, Witten-Reshetikhin-Turaev invariants.

The first author was supported in part by NSF. 


\section{The Colored $\mathfrak{s l}_{3}$ Jones polynomial of the trefoil}

In his seminal paper Jon87, Jones introduced the Jones polynomial of a knot $K$ in 3 -space. The Jones polynomial is a Laurent polynomial in a variable $q$ with integer coefficients, which can be generalized to an invariant $J_{K, V}(q) \in \mathbb{Z}\left[q^{ \pm 1}\right]$ of a (0-framed) knot $K$ colored by a representation $V$ of a simple Lie algebra $\mathfrak{g}$, and normalized to be 1 at the unknot. The definition of $J_{K, V}(q)$ uses the machinery of quantum groups and may be found in [Tur88, Tur94] and also in [Jan96.

Concrete formulas for the colored Jones polynomial $J_{K, V}(q)$ are hard to find in the case of higher rank Lie algebras, and for good reasons. For torus knots $T$, Jones and Rosso gave a formula for $J_{T, V}(q)$ which involves a plethysm map of $V$, unknown in general. Our goal is to give an explicit formula for the second plethysm of representations of $\mathfrak{s l}_{3}$ and consequently to give a formula for the $\mathfrak{s l}_{3}$ colored Jones polynomial of the trefoil. To state our results, let $V_{n_{1}, n_{2}}$ denote the irreducible representation of $\mathfrak{s l}_{3}$ with highest weight,

$$
\lambda=n_{1} \omega_{1}+n_{2} \omega_{2},
$$

where $n_{1}, n_{2}$ are nonnegative integers and $\omega_{1}, \omega_{2}$ are the fundamental weights of $\mathfrak{s l}_{3}$ dual to the simple roots $\alpha_{1}, \alpha_{2}$. In coordinates, we have

$\alpha_{1}=(1,-1,0), \quad \alpha_{2}=(0,1,-1), \quad \omega_{1}=\frac{1}{3}\left(2 \alpha_{1}+\alpha_{2}\right), \quad \omega_{2}=\frac{1}{3}\left(\alpha_{1}+2 \alpha_{2}\right)$.

The quantum integer $[n]$, the quantum dimension $d_{n_{1}, n_{2}}$ and the twist parameter $\theta_{n_{1}, n_{2}}$ of $V_{n_{1}, n_{2}}$ are defined by

$$
\begin{aligned}
{[n] } & =\frac{q^{\frac{n}{2}}-q^{-\frac{n}{2}}}{q^{\frac{1}{2}}-q^{-\frac{1}{2}}}, \\
d_{n_{1}, n_{2}} & =\frac{\left[n_{1}+1\right]\left[n_{2}+1\right]\left[n_{1}+n_{2}+2\right]}{[2]}, \\
\theta_{n_{1}, n_{2}} & =q^{\frac{1}{3}\left(n_{1}^{2}+n_{1} n_{2}+n_{2}^{2}\right)+n_{1}+n_{2}} .
\end{aligned}
$$

Let $T(m, n)$ denote the torus knot associated to a pair of coprime natural numbers $m, n$, and let $J_{T(m, n), n_{1}, n_{2}}(q)$ denote the $\mathfrak{s l}_{3}$ colored Jones polynomial of the torus knot $T(m, n)$ colored by $V_{n_{1}, n_{2}}$.

Theorem 2.1. For all odd natural numbers $n$ we have

$$
\begin{gathered}
J_{T(2, n), n_{1}, n_{2}}(q) \\
=\frac{\theta_{n_{1}, n_{2}}^{-2 n}}{d_{n_{1}, n_{2}}}\left(\sum_{l=0}^{\min \left\{n_{1}, n_{2}\right\}} \sum_{k=0}^{n_{1}-l}(-1)^{k} d_{2 n_{1}-2 k-2 l, 2 n_{2}+k-2 l} \theta_{2 n_{1}-2 k-2 l, 2 n_{2}+k-2 l}^{\frac{n}{2}}\right. \\
+\sum_{l=0}^{\min \left\{n_{1}, n_{2}\right\}} \sum_{k=0}^{n_{2}-l}(-1)^{k} d_{2 n_{1}+k-2 l, 2 n_{2}-2 k-2 l} \theta_{2 n_{1}+k-2 l, 2 n_{2}-2 k-2 l}^{\frac{n}{2}} \\
\left.-\sum_{l=0}^{\min \left\{n_{1}, n_{2}\right\}} d_{2 n_{1}-2 l, 2 n_{2}-2 l} \theta_{2 n_{1}-2 l, 2 n_{2}-2 l}^{\frac{n}{2}}\right) .
\end{gathered}
$$

Theorem 2.1 can be used to answer several problems.

- We can verify the $\mathfrak{s l}_{3}$-Degree Conjecture of the colored Jones polynomial for the trefoil; see $[\mathrm{GV}$. Explicitly, we can compute the lowest degree 
$\delta_{T(2, n), n_{1}, n_{2}}^{*}$ and the highest degree $\delta_{T(2, n), n_{1}, n_{2}}$ of the Laurent polynomial $J_{T(2, n), n_{1}, n_{2}}(q)$ as follows:

$$
\begin{aligned}
& \delta_{T(2, n), n_{1}, n_{2}}^{*}= \begin{cases}-\frac{n}{2} n_{1}^{2}-\frac{n}{2} n_{2}^{2}-n n_{1} n_{2}-\frac{3 n}{2} n_{1}-\left(\frac{5 n}{2}-2\right) n_{2} & \text { if } n_{1} \geq n_{2}, \\
-\frac{n}{2} n_{1}^{2}-\frac{n}{2} n_{2}^{2}-n n_{1} n_{2}-\frac{3 n}{2} n_{2}-\left(\frac{5 n}{2}-2\right) n_{1} & \text { if } n_{1}<n_{2},\end{cases} \\
& \delta_{T(2, n), n_{1}, n_{2}}=-(n-1)\left(n_{1}+n_{2}\right) .
\end{aligned}
$$

The above formula verifies that the degree, restricted to each Kostant chamber, is a quadratic quasi-polynomial.

- We can efficiently compute the Witten-Reshetikhin-Turaev invariant of the Poincaré sphere, complementing calculations of Lawrence Law03.

- We can guess an explicit Groebner basis for the ideal of recursion relations of the 2-variable $q$-holonomic sequence $J_{T(2,3), n_{1}, n_{2}}(q)$; see [GK10].

Remark 2.2. An alternative formula for the $\mathfrak{s l}_{3}$ colored Jones polynomial of $T(2,3)$ is given by Lawrence in Law03. Lawrence's formula is derived from the theory of Quantum Groups and cannot generalize to the case of $T(2, n)$ torus knots. In contrast, the plethysm formula of Theorem 2.4 below can be generalized to a formula for $\psi_{m}\left(V_{\lambda}\right)$ which allows for an efficient formula of the $\mathfrak{s l}_{3}$ colored Jones polynomial of all torus knots. Additional generalizations are possible for all simple Lie algebras; see $\mathrm{GV}$.

Remark 2.3. Theorem 2.1 gives an efficient computation of the $\mathfrak{s l}_{3}$ colored Jones polynomial of the $3_{1}, 5_{1}, 7_{1}$ and $9_{1}$ knots in the Rolfsen notation. In low weights, our answer agrees with the independent computation given by the entirely different methods of the KnotAtlas; see [BN05]. This is a consistency check which simultaneously validates the formulas of Theorem 2.1 and the data of the KnotAtlas.

2.1. An $\mathfrak{s l}_{3}$ plethysm formula. As mentioned above, Theorem 2.1 follows from the Rosso-Jones formula for the colored Jones polynomial of torus knots and the following plethysm computation. Let $\psi_{m}$ denote the $m$-plethysm operation.

Theorem 2.4. For $\lambda$ as in Equation (1) we have

$$
\begin{aligned}
& \psi_{2}\left(V_{\lambda}\right)=\sum_{l=0}^{\min \left\{n_{1}, n_{2}\right\}} \sum_{k=0}^{n_{1}-l}(-1)^{k} V_{2 \lambda-k \alpha_{1}-2 l\left(\alpha_{1}+\alpha_{2}\right)} \\
& +\sum_{l=0}^{\min \left\{n_{1}, n_{2}\right\}} \sum_{k=0}^{n_{2}-l}(-1)^{k} V_{2 \lambda-k \alpha_{2}-2 l\left(\alpha_{1}+\alpha_{2}\right)} \\
& \min \left\{n_{1}, n_{2}\right\} \\
& \text { - } \sum_{l=0} V_{2 \lambda-2 l\left(\alpha_{1}+\alpha_{2}\right)} \text {. }
\end{aligned}
$$

\section{The Rosso-Jones FORMula}

The polynomial invariant $J_{K, V}(q)$ of a knot $K$ colored by the representation $V$ of a simple Lie algebra is difficult to compute from its Quantum Group definition even when $K=4_{1}$ and $\mathfrak{g}=\mathfrak{s l}_{3}$. Although it is a finite multi-dimensional sum, a practical computation seems out of reach. Fortunately, there is a class of knots whose quantum group invariant has a simple enough formula that allows us to extract its $q$-degree. This is the class of torus knots $T(m, n)$ where $m, n$ are coprime natural numbers. The simple formula is due to Rosso and Jones and is also studied 
by the second named author, RJ93, Mor95. Let $d_{\lambda}$ denote the quantum dimension of the representation $V_{\lambda}$ and let $\theta_{\lambda}$ be the eigenvalue of the twist operator on the representation $V_{\lambda} \cdot d_{\lambda}$ and $\theta_{\lambda}$ are given by

$$
\begin{aligned}
& d_{\lambda}=\prod_{\alpha>0} \frac{[(\lambda+\rho, \alpha)]}{[(\rho, \alpha)]}, \\
& \theta_{\lambda}=q^{\frac{1}{2}(\lambda, \lambda+2 \rho)},
\end{aligned}
$$

where $\alpha$ belongs to the set of positive roots, $\rho=\frac{1}{2} \sum_{\alpha>0} \alpha$ is half the sum of the positive roots and $(\cdot, \cdot)$ denotes the $\mathfrak{g}$-invariant inner product on the dual of the Cartan algebra (normalized so that the longest root has length $\sqrt{2}$ ). When $\mathfrak{g}=\mathfrak{s l}_{3}$ and $\lambda$ is given by (10), then the quantum dimension and the twist parameter coincide with (3) and (4). For a natural number $m$, consider the $m$-Adams operation $\psi_{m}$ on representations. It is given by (see [FH91, Mac95])

$$
\psi_{m}\left(V_{\lambda}\right)=\sum_{\mu \in S_{\lambda, m}} c_{\lambda, m}^{\mu} V_{\mu}
$$

where $c_{\lambda, m}^{\mu}$ are nonzero integers. The Rosso-Jones formula is the following (see [RJ93]):

$$
J_{T(m, n), \lambda}(q)=\frac{\theta_{\lambda}^{-m n}}{d_{\lambda}} \sum_{\mu \in S_{\lambda, m}} c_{\lambda, m}^{\mu} d_{\mu} \theta_{\mu}^{\frac{n}{m}} .
$$

For a related discussion, see also [MM08].

\section{SCHUR FUNCTIONS IN $\mathfrak{s l}_{3}$}

4.1. A review of Schur functions. Let us recall some well-known properties of Schur functions and their relation to the character of irreducible representations of $\mathfrak{s l}_{N}$, which can be found in [Mac95, FH91]. For a partition $\lambda$ with parts $\lambda_{1} \geq \lambda_{2} \geq$ $\ldots \geq \lambda_{k} \geq 0$, let $s_{\lambda_{1}, \ldots, \lambda_{k}}\left(x_{1}, \ldots, x_{N}\right)$ denote the corresponding Schur function. A partition $\lambda=\left(\lambda_{1}, \ldots, \lambda_{k}\right)$ will be depicted as an arrangement of boxes as follows (for $\lambda=(4,2,1))$ :

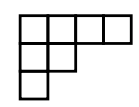

If $\omega_{i}$ denote the fundamental weights of $\mathfrak{s l}_{N}$ and $n_{i}$ are nonnegative integers for $i=1, \ldots, N-1$, and $\lambda=\left(\sum_{i=1}^{N-1} n_{i}, \sum_{i=2}^{N-1} n_{i}, \ldots, \sum_{i=N-1}^{N-1} n_{i}\right)$, then

$$
\operatorname{character}\left(V_{\sum_{i=1}^{N-1} n_{i} \omega_{i}}\right)=s_{\lambda}\left(x_{1}, \ldots, x_{N}\right) .
$$

For $\lambda=(4,2,1)$ we then have $\left(n_{1}, n_{2}, n_{3}\right)=(2,1,1)$.

The plethysm operation $\psi_{m}$ is defined by

$$
\psi_{m}\left(s_{\lambda}\left(x_{1}, \ldots, x_{N}\right)\right)=s_{\lambda}\left(x_{1}^{m}, \ldots, x_{N}^{m}\right) .
$$

Note that $s_{1}=x_{1}+\cdots+x_{N}$ and $\psi_{2}\left(s_{1}\right)=s_{2}-s_{1,1}$.

In $\mathfrak{s l}_{N}$ the irreducible modules correspond to partitions $\lambda$ with at most $N$ parts. The decomposition of $\psi_{m}\left(V_{\lambda}\right)$ into irreducibles needed for the invariant of the $(m, n)$ torus knot is given by the corresponding expansion of the symmetric function $\psi_{m}\left(s_{\lambda}\right)$ as a linear combination of Schur functions. 
When $N=3$ the Schur function $s_{\lambda}$ vanishes where $\lambda$ has more than 3 parts and satisfies $s_{a, b, c}=s_{a+1, b+1, c+1}$. Then $s_{a, b, c}=s_{a-c, b-c}$, so we need only consider partitions with at most 2 parts. All the same, it will be convenient to use 3 parts in what follows.

4.2. A reformulation of Theorem 2.4. The goal of this section is to give a formula for $\psi_{2}\left(s_{m_{1}, m_{2}}\right)$ as a linear combination of Schur functions, assuming that $N=3$.

Definition 4.1. For $m_{1} \geq m_{2} \geq 0$, let $D\left(m_{1}, m_{2}\right) \subset \mathbb{N}^{3}$ denote the set of tuples $(a, b, c)$ that satisfy

- $a+b+c=2 m_{1}+2 m_{2}, 2 m_{1} \geq a \geq b \geq c \geq 0, a \geq 2 m_{2} \geq c$;

- if $b \geq 2 m_{2}$, then $c \equiv 0 \bmod 2$;

- if $b \leq 2 m_{2}$, then $a \equiv 0 \bmod 2$.

When $m_{1}<m_{2}$, we define $D\left(m_{1}, m_{2}\right)$ to be the empty set.

Theorem 4.2. In $\mathfrak{s l}_{3}$ for all $m_{1} \geq m_{2}$ we have

$$
\psi_{2}\left(s_{m_{1}, m_{2}}\right)=\sum_{(a, b, c) \in D\left(m_{1}, m_{2}\right)}(-1)^{b} s_{a, b, c} .
$$

It is interesting to note that the coefficient of every Schur function in the expansion of $\psi_{2}\left(s_{m_{1}, m_{2}}\right)$ is $0, \pm 1$. The same feature proves to be the case for $\psi_{2}\left(s_{m_{1}, m_{2}}\right)$ in the general case of $\mathfrak{s l}_{N}$, noted in Subsection 5.1 .

4.3. Theorem 4.2 implies Theorem 2.4. Since $V_{n_{1} \omega_{1}+n_{2} \omega_{2}}^{*}=V_{n_{2} \omega_{1}+n_{1} \omega_{2}}$ and $J_{K, V^{*}}(q)=J_{K, V}(1 / q)$, it suffices to prove Theorem 2.4 when $n_{1} \geq n_{2}$. Equation (11) for $N=3$ implies that

$$
\operatorname{character}\left(V_{n_{1} \omega_{1}+n_{2} \omega_{2}}\right)=s_{n_{1}+n_{2}, n_{2}}\left(x_{1}, x_{2}, x_{3}\right) .
$$

Fix nonnegative integers $n_{1}$ and $n_{2}$ and set $\left(m_{1}, m_{2}\right)=\left(n_{1}+n_{2}, n_{2}\right)$ in Theorem 4.2 ,

We can parametrise a tuple $(a, b, c) \in D\left(m_{1}, m_{2}\right)$ that satisfies $b \geq 2 m_{2}$ by setting $b=2 m_{2}+k, c=2 l$, to get $a=2 m_{1}-k-2 l$, satisfying the inequalities $k, l \geq 0, k \leq m_{1}-m_{2}-l, l \leq m_{2}, m_{1}-m_{2}$. Likewise, we can parametrise a tuple $(a, b, c) \in D\left(m_{1}, m_{2}\right)$ that satisfies $b \leq 2 m_{2}$ by setting $b=2 m_{2}-k, a=2 m_{1}-2 l$ to get $c=2 l+k$, satisfying $k, l \geq 0, k \leq m_{2}-l, l \leq m_{2}, m_{1}-m_{2}$. Thus Theorem 4.2 implies the formula of Theorem 2.4 .

4.4. A reformulation of Theorem 4.2, To establish Theorem 4.2 we first prove Theorem 4.3

Theorem 4.3. For $m_{1} \geq m_{2}$ we have

$$
\begin{aligned}
\left(\sum_{(a, b, c) \in D\left(m_{1}, m_{2}\right)}(-1)^{b} s_{a, b, c}\right) \psi_{2}\left(s_{1}\right)= & \sum_{\left(a^{\prime}, b^{\prime}, c^{\prime}\right) \in D\left(m_{1}+1, m_{2}\right)}(-1)^{b^{\prime}} s_{a^{\prime}, b^{\prime}, c^{\prime}} \\
& +\sum_{\left(a^{\prime}, b^{\prime}, c^{\prime}\right) \in D\left(m_{1}, m_{2}+1\right)}(-1)^{b^{\prime}} s_{a^{\prime}, b^{\prime}, c^{\prime}} \\
& +\sum_{\substack{\left(a^{\prime}, b^{\prime}, c^{\prime}\right) \in D\left(m_{1}-1, m_{2}-1\right) \\
m_{2}>0}}(-1)^{b^{\prime}} s_{a^{\prime}, b^{\prime}, c^{\prime}} .
\end{aligned}
$$


In the proof of Theorem 4.2 we will need the following special cases of the Littlewood-Richardson rule adapted to $\mathfrak{s l}_{3}$, bearing in mind that Schur functions for partitions with more than 3 parts are 0 in this case; see Mac95. In the next lemma and below, we will use the convention that $s_{a_{1}, a_{2}, a_{3}}=0$ unless $a_{1} \geq a_{2} \geq a_{3}$. Furthermore, the notation $\left.s_{a, b, c}\right|_{a>b}$ (resp. $\left.s_{a, b, c}\right|_{a=b}$ ) means $s_{a, b, c}$ when $a>b$ (resp. $a=b)$ and zero otherwise.

Lemma 4.4. In $\mathfrak{s l}_{3}$ we have

$$
\begin{aligned}
s_{a, b, c} s_{2}= & s_{a+2, b, c}+s_{a, b+2, c}+s_{a, b, c+2}+\left.s_{a+1, b+1, c}\right|_{a>b} \\
& +s_{a+1, b, c+1}+\left.s_{a, b+1, c+1}\right|_{b>c} \\
s_{a, b, c} s_{1,1}= & s_{a+1, b+1, c}+s_{a+1, b, c+1}+s_{a, b+1, c+1} \\
s_{m_{1}, m_{2}} s_{1}= & s_{m_{1}+1, m_{2}}+s_{m_{1}, m_{2}+1}+s_{m_{1}, m_{2}, 1}
\end{aligned}
$$

Corollary 4.5. For $a \geq b \geq c \geq 0$ we have

$s_{a, b, c}\left(s_{2}-s_{1,1}\right)=s_{a+2, b, c}+s_{a, b+2, c}+s_{a, b, c+2}-\left.s_{a+1, b+1, c}\right|_{a=b}-\left.s_{a, b+1, c+1}\right|_{b=c}$.

Corollary 4.6. Since $\psi_{2}$ is a ring homomorphism and $\psi_{2}\left(s_{1}\right)=s_{2}-s_{1,1}$, we have

$$
\begin{aligned}
& \psi_{2}\left(s_{m_{1}, m_{2}}\right)\left(s_{2}-s_{1,1}\right)=\psi_{2}\left(s_{m_{1}, m_{2}}\right) \psi_{2}\left(s_{1}\right)=\psi_{2}\left(s_{m_{1}, m_{2}} s_{1}\right) \\
& \quad= \begin{cases}\psi_{2}\left(s_{m_{1}+1, m_{2}}\right)+\psi_{2}\left(s_{m_{1}, m_{2}+1}\right)+\psi_{2}\left(s_{m_{1}, m_{2}, 1}\right) & \text { if } m_{1}>m_{2}>0, \\
\psi_{2}\left(s_{m_{1}+1, m_{2}}\right)+\psi_{2}\left(s_{m_{1}, m_{2}+1}\right) & \text { if } m_{1}>m_{2}=0 .\end{cases}
\end{aligned}
$$

4.5. Theorem 4.3 implies Theorem 4.2. We deduce Theorem 4.2 from Theorem 4.3 by induction on $m_{2}$.

When $m_{2}=0$ we have $(a, b, c) \in D\left(m_{1}, 0\right)$ iff $c=0, a+b=2 m_{1}, a \geq b \geq 0$. It is known (for example, CGR84, Eqn. 2.30]) that

$$
\psi_{2}\left(s_{m}\right)=\sum_{k=0}^{m}(-1)^{k} s_{2 m-k, k} .
$$

This establishes Theorem 4.2 for $m_{2}=0$.

Theorem 4.3 gives

$$
\begin{aligned}
\psi_{2}\left(s_{m_{1}, m_{2}}\right) \psi_{2}\left(s_{1}\right)= & \psi_{2}\left(s_{m_{1}+1, m_{2}}\right) \\
& +\sum_{\left(a^{\prime}, b^{\prime}, c^{\prime}\right) \in D\left(m_{1}, m_{2}+1\right)}(-1)^{b^{\prime}} s_{a^{\prime}, b^{\prime}, c^{\prime}}+\psi_{2}\left(s_{m_{1}-1, m_{2}-1}\right)
\end{aligned}
$$

by induction on $m_{2}$.

Corollary 4.6 then shows that

$$
\psi_{2}\left(s_{m_{1}, m_{2}+1}\right)=\sum_{\left(a^{\prime}, b^{\prime}, c^{\prime}\right) \in D\left(m_{1}, m_{2}+1\right)}(-1)^{b^{\prime}} s_{a^{\prime}, b^{\prime}, c^{\prime}},
$$

which completes the induction step.

4.6. Proof of Theorem 4.3. To prove Theorem 4.3 we sum both sides of the equation in Corollary 4.5 over $(a, b, c) \in D\left(m_{1}, m_{2}\right)$, using the following lemma. 
Lemma 4.7. Suppose that $m_{1}>m_{2} \geq 0$. Then

$$
\begin{aligned}
& \sum_{(a, b, c) \in D\left(m_{1}, m_{2}\right)}(-1)^{b} s_{a+2, b, c}=\sum_{\substack{\left(a^{\prime}, b^{\prime}, c^{\prime}\right) \in D\left(m_{1}+1, m_{2}\right) \\
a^{\prime} \neq b^{\prime}, a^{\prime} \neq 2 m_{2}}}(-1)^{b^{\prime}} s_{a^{\prime}, b^{\prime}, c^{\prime}}, \\
& \sum_{(a, b, c) \in D\left(m_{1}, m_{2}\right)}(-1)^{b} s_{a, b+2, c}=\sum_{\substack{\left(a^{\prime}, b^{\prime}, c^{\prime}\right) \in D\left(m_{1}, m_{2}+1\right) \\
b^{\prime} \neq c^{\prime}, c^{\prime} \neq 2 m_{2}+2}}(-1)^{b^{\prime}} s_{a^{\prime}, b^{\prime}, c^{\prime}} \\
& +\sum_{\substack{\left(a^{\prime}, b^{\prime}, c^{\prime}\right) \in D\left(m_{1}+1, m_{2}\right) \\
a^{\prime}=2 m_{2}, b^{\prime} \neq c^{\prime}}}(-1)^{b^{\prime}} s_{a^{\prime}, b^{\prime}, c^{\prime}}, \\
& \sum_{(a, b, c) \in D\left(m_{1}, m_{2}\right)}(-1)^{b} s_{a, b, c+2}=\sum_{\substack{\left(a^{\prime}, b^{\prime}, c^{\prime}\right) \in D\left(m_{1}-1, m_{2}-1\right) \\
m_{2}>0}}(-1)^{b^{\prime}} s_{a^{\prime}, b^{\prime}, c^{\prime}} \\
& +\sum_{\substack{\left(a^{\prime}, b^{\prime}, c^{\prime}\right) \in D\left(m_{1}, m_{2}+1\right) \\
c^{\prime}=2 m_{2}+2}}(-1)^{b^{\prime}} s_{a^{\prime}, b^{\prime}, c^{\prime}}, \\
& \sum_{(a, b, c) \in D\left(m_{1}, m_{2}\right)}(-1)^{b+1} s_{a+1, b+1, c}=\sum_{\substack{\left(a^{\prime}, b^{\prime}, c^{\prime}\right) \in D\left(m_{1}+1, m_{2}\right) \\
a^{\prime}=b^{\prime}, a^{\prime} \neq 2 m_{2}, b^{\prime} \neq c^{\prime}}}(-1)^{b^{\prime}} s_{a^{\prime}, b^{\prime}, c^{\prime}}, \\
& \sum_{\substack{(a, b, c) \in D\left(m_{1}, m_{2}\right) \\
b=c}}(-1)^{b+1} s_{a, b+1, c+1}=\sum_{\substack{\left(a^{\prime}, b^{\prime}, c^{\prime}\right) \in D\left(m_{1}, m_{2}+1\right) \\
b^{\prime}=c^{\prime}, c^{\prime} \neq 2 m_{2}+2}}(-1)^{b^{\prime}} s_{a^{\prime}, b^{\prime}, c^{\prime}} \\
& +\sum_{\substack{\left(a^{\prime}, b^{\prime}, c^{\prime}\right) \in D\left(m_{1}+1, m_{2}\right) \\
a^{\prime}=2 m_{2}, b^{\prime}=c^{\prime}}}(-1)^{b^{\prime}} s_{a^{\prime}, b^{\prime}, c^{\prime}} .
\end{aligned}
$$

The total sum of the left hand sides of the equations in Lemma 4.7 is then the left hand side of the equation in Theorem 4.3, while the terms on the right hand sides make up the right hand side of Theorem 4.3 .

4.7. Proof of Lemma 4.7. For each of the five equations we provide a bijective transformation carrying $(a, b, c) \in D\left(m_{1}, m_{2}\right)$ with the restrictions shown to $\left(a^{\prime}, b^{\prime}, c^{\prime}\right)$ satisfying the conditions on the right hand sides.

We make repeated use of the parity rules to ensure that inequalities force a difference of at least 2 . With the exception of a couple of less obvious cases we omit proofs that the individual parity rules for $\left(a^{\prime}, b^{\prime}, c^{\prime}\right)$ are satisfied, as they generally follow readily from those for $(a, b, c)$ and vice versa. Equally the sum $a^{\prime}+b^{\prime}+c^{\prime}$ is always obviously correct.

Proof. For Equation (12), put $a^{\prime}=a+2, b^{\prime}=b, c^{\prime}=c$. Let $(a, b, c) \in D\left(m_{1}, m_{2}\right)$. Then $2 m_{2}+2 \geq a^{\prime}>b^{\prime} \geq c^{\prime} \geq 0$, and $a^{\prime}>2 m_{2} \geq c^{\prime}$. Then $\left(a^{\prime}, b^{\prime}, c^{\prime}\right) \in$ $D\left(m_{1}+1, m_{2}\right)$, with $a^{\prime} \neq b^{\prime}$ and $a^{\prime} \neq 2 m_{2}$.

Conversely suppose that $\left(a^{\prime}, b^{\prime}, c^{\prime}\right) \in D\left(m_{1}+1, m_{2}\right)$, with $a^{\prime}>b^{\prime}$ and $a^{\prime}>2 m_{2}$. By the parity rules, if $b^{\prime} \leq 2 m_{2}$, then $a^{\prime} \equiv 0 \bmod 2$, so $a^{\prime} \geq 2 m_{2}+2 \geq b^{\prime}+2$. If $b^{\prime}>2 m_{2}$, then $a^{\prime} \equiv b^{\prime} \bmod 2$, so $a^{\prime} \geq b^{\prime}+2>2 m_{2}+2$. In any case $2 m_{1} \geq$ $a^{\prime}-2 \geq b^{\prime} \geq c^{\prime} \geq 0$, and $a^{\prime}-2 \geq 2 m_{2} \geq c^{\prime}$. Then $(a, b, c) \in D\left(m_{1}, m_{2}\right)$. This proves Equation (12). 
For Equation (13), put $a^{\prime}=a, b^{\prime}=b+2, c^{\prime}=c$. Let $(a, b, c) \in D\left(m_{1}, m_{2}\right)$ with $a \geq b+2$. If $a=2 m_{2}$, then $2 m_{1}+2 \geq a^{\prime} \geq b^{\prime}>c^{\prime} \geq 0$ and $a^{\prime} \geq 2 m_{2} \geq c^{\prime}$. Then $\left(a^{\prime}, b^{\prime}, c^{\prime}\right) \in D\left(m_{1}+1, m_{2}\right)$, with $a^{\prime}=2 m_{2}, b^{\prime}>c^{\prime}$. Otherwise $a>2 m_{2}$. If $b \geq 2 m_{2}$, then $a \geq b+2 \geq 2 m_{2}+2$, while if $b<2 m_{2}$, then $a \equiv 0 \bmod 2$ by the parity rules, so that $a \geq 2 m_{2}+2$. Hence $2 m_{1} \geq a^{\prime} \geq b^{\prime}>c^{\prime} \geq 0$ and $a^{\prime} \geq 2 m_{2}+2 \geq c^{\prime}$. In this case we check the parity rules explicitly. Here $b^{\prime} \geq 2 m_{2}+2 \Longrightarrow b \geq 2 m_{2} \Longrightarrow$ $c^{\prime} \equiv c \equiv 0 \bmod 2$ and $b^{\prime} \leq 2 m_{2}+2 \Longrightarrow b \leq 2 m_{2} \Longrightarrow a^{\prime} \equiv a \equiv 0 \bmod 2$. So $\left(a^{\prime}, b^{\prime}, c^{\prime}\right) \in D\left(m_{1}, m_{2}+1\right)$ with $b^{\prime}>c^{\prime}$ and $c^{\prime}<2 m_{2}+2$.

Conversely suppose that $\left(a^{\prime}, b^{\prime}, c^{\prime}\right) \in D\left(m_{1}, m_{2}+1\right)$ with $b^{\prime}>c^{\prime}$ and $c^{\prime}<2 m_{2}+2$. If $b^{\prime} \geq 2 m_{2}+2$, then $c^{\prime} \equiv 0 \bmod 2$, so $c^{\prime} \leq 2 m_{2} \leq b^{\prime}-2$; and if $b^{\prime}<2 m_{2}+2$, then $b^{\prime} \equiv c^{\prime} \bmod 2$ and $c^{\prime} \leq b^{\prime}-2<2 m_{2}$. Hence $2 m_{1} \geq a^{\prime} \geq b^{\prime}-2 \geq c^{\prime} \geq 0$ and $a^{\prime}>2 m_{2}$. A parity check as above shows that then $(a, b, c) \in D\left(m_{1}, m_{2}\right)$ with $a=a^{\prime} \geq b^{\prime}=b+2$ and $a>2 m_{2}$.

Finally suppose that $\left(a^{\prime}, b^{\prime}, c^{\prime}\right) \in D\left(m_{1}+1, m_{2}\right)$, with $a^{\prime}=2 m_{2}, b^{\prime}>c^{\prime}$. Then $b^{\prime} \equiv c^{\prime} \bmod 2$, so $b^{\prime}-2 \geq c^{\prime}$, and $a^{\prime}=2 m_{2} \geq c^{\prime}$, again giving $(a, b, c) \in D\left(m_{1}, m_{2}\right)$ with $a=2 m_{2} \geq b+2$. This proves Equation (13).

For Equation (14), put $a^{\prime}=a, b^{\prime}=b, c^{\prime}=c+2$ when $c=2 m_{2}$, and $a^{\prime}=$ $a-2, b^{\prime}=b-2, c^{\prime}=c$ otherwise. In either case $s_{a, b, c+2}=s_{a^{\prime}, b^{\prime}, c^{\prime}}$ since we are working in $\mathfrak{s l}_{3}$. Let $(a, b, c) \in D\left(m_{1}, m_{2}\right)$ with $b \geq c+2$. If $c=2 m_{2}$, then $2 m_{1} \geq a^{\prime} \geq b^{\prime} \geq 2 m_{2}+2=c^{\prime} \geq 0$, and $\left(a^{\prime}, b^{\prime}, c^{\prime}\right) \in D\left(m_{1}, m_{2}+1\right)$ with $c^{\prime}=2 m_{2}+2$. Otherwise $c<2 m_{2} \neq 0$. If $b \leq 2 m_{2}$, then $c \leq 2 m_{2}-2$. If $b>2 m_{2}$, then $c \equiv 0 \bmod 2$ by the parity rules, giving again $c \leq 2 m_{2}-2$. Then $2 m_{1}-2 \geq a-2 \geq b-2 \geq c \geq 0$ and $a-2 \geq 2 m_{2}-2 \geq c$. So $\left(a^{\prime}, b^{\prime}, c^{\prime}\right) \in D\left(m_{1}-1, m_{2}-1\right)$.

Conversely let $\left(a^{\prime}, b^{\prime}, c^{\prime}\right) \in D\left(m_{1}-1, m_{2}-1\right)$, with $m_{2} \neq 0$. Then $2 m_{1} \geq$ $a^{\prime}+2 \geq b^{\prime}+2 \geq c^{\prime} \geq 0$ and $a^{\prime} \geq 2 m_{2}-2 \geq c^{\prime}$, so $a^{\prime}+2 \geq 2 m_{2}>c^{\prime}$. Hence $(a, b, c) \in D\left(m_{1}, m_{2}\right)$ with $c \neq 2 m_{2}$.

Finally, suppose that $\left(a^{\prime}, b^{\prime}, c^{\prime}\right) \in D\left(m_{1}, m_{2}+1\right)$ with $c^{\prime}=2 m_{2}+2$. Then $2 m_{1} \geq a^{\prime} \geq b^{\prime} \geq 2 m_{2}=c^{\prime}-2 \geq 0$ so that $\left(a^{\prime}, b^{\prime}, c^{\prime}-2\right)=(a, b, c) \in D\left(m_{1}, m_{2}\right)$ with $c=2 m_{2}$. This proves Equation (14).

For Equation (15), put $a^{\prime}=a+1, b^{\prime}=b+1, c^{\prime}=c$. Let $(a, b, c) \in D\left(m_{1}, m_{2}\right)$ with $a=b$. Then $2 m_{1}+2 \geq a^{\prime} \geq b^{\prime} \geq c^{\prime} \geq 0$ and $a^{\prime}>a \geq 2 m_{2} \geq c^{\prime} \geq 0$. Since $b^{\prime}=a^{\prime}>2 m_{2}$ and $c^{\prime} \equiv 0 \bmod 2$ the parity rules are satisfied, and $\left(a^{\prime}, b^{\prime}, c^{\prime}\right) \in$ $D\left(m_{1}+1, m_{2}\right)$ with $a^{\prime}=b^{\prime}, a^{\prime}>2 m_{2}, b^{\prime} \neq c^{\prime}$.

Conversely let $\left(a^{\prime}, b^{\prime}, c^{\prime}\right) \in D\left(m_{1}+1, m_{2}\right)$ with $a^{\prime}=b^{\prime}, a^{\prime}>2 m_{2}, b^{\prime} \neq c^{\prime}$. Now $2 a^{\prime} \leq a^{\prime}+b^{\prime}+c^{\prime}=2 m_{1}+2 m_{2}+2 \leq 4 m_{1}$, since $m_{2}<m_{1}$. Then $2 m_{1}>a^{\prime}-1 \geq$ $b^{\prime}-1 \geq c^{\prime} \geq 0$ and $a^{\prime}-1 \geq 2 m_{2} \geq c^{\prime}$. Hence $(a, b, c) \in D\left(m_{1}, m_{2}\right)$ with $a=\bar{b}$. This proves Equation (14).

For Equation (16), put $a^{\prime}=a, b^{\prime}=b+1, c^{\prime}=c+1$. Let $(a, b, c) \in D\left(m_{1}, m_{2}\right)$ with $a>b=c$. If $a=2 m_{2}$, then $2 m_{1}+2>a^{\prime} \geq b^{\prime} \geq c^{\prime} \geq 0$ and $a^{\prime}=2 m_{2} \geq c^{\prime}$. Hence $\left(a^{\prime}, b^{\prime}, c^{\prime}\right) \in D\left(m_{1}+1, m_{2}\right)$ with $a^{\prime}=2 m_{2}, b^{\prime}=c^{\prime}$. Otherwise $a>2 m_{2}$, and $a^{\prime}=a \geq 2 m_{2}+2$, since $b=c$, while $2 m_{2}+2 \geq c+2>c^{\prime}$. We have also $2 m_{1} \geq a^{\prime} \geq b^{\prime} \geq c^{\prime} \geq 0$. Hence $\left(a^{\prime}, b^{\prime}, c^{\prime}\right) \in D\left(m_{1}, m_{2}+1\right)$ with $b^{\prime}=c^{\prime}, c^{\prime} \neq 2 m_{2}+2$.

Conversely suppose that $\left(a^{\prime}, b^{\prime}, c^{\prime}\right) \in D\left(m_{1}, m_{2}+1\right)$ with $b^{\prime}=c^{\prime}, c^{\prime}<2 m_{2}+2$. Now $a^{\prime}+2 c^{\prime}=2 m_{1}+2 m_{2}+2$ and $a^{\prime} \leq 2 m_{1}$, so $c^{\prime}>0$. Hence $2 m_{1} \geq a^{\prime}>b^{\prime}-1 \geq$ $c^{\prime}-1 \geq 0$ and $a^{\prime}>2 m_{2} \geq c^{\prime}-1$. Then $(a, b, c) \in D\left(m_{1}, m_{2}\right)$ with $a>b=c$ and $a>2 m_{2}$.

Finally if $\left(a^{\prime}, b^{\prime}, c^{\prime}\right) \in D\left(m_{1}+1, m_{2}\right)$ with $a^{\prime}=2 m_{2}, b^{\prime}=c^{\prime}$, then $b^{\prime}=c^{\prime}=$ $m_{1}+1>0$ and $(a, b, c) \in D\left(m_{1}, m_{2}\right)$ with $a=2 m_{2}>b=c$. 


\section{A proof of Theorem 4.2 using CArini-Remmel's Work}

5.1. A review of Theorem 5 of CR98. In this section we give an alternative proof of Theorem 4.2 using the work of Carini and Remmel [CR98]. In Theorem 5 of [CR98], Carini and Remmel give the expansion of the plethysm $\psi_{2}\left(s_{a, b}\right)$ for the Schur function of a 2-row partition of $n=a+b$ in terms of Schur functions $s_{\lambda}$, where $\lambda$ runs through partitions of $2 n$ with at most 4 parts. In this expansion each $s_{\lambda}$ has coefficient $0, \pm 1$, depending on the parities of the parts of $\lambda$ and some linear inequalities.

In their paper Carini and Remmel use the opposite convention to Macdonald, so that they take $0 \leq a \leq b$ for the given partition of $n=a+b$ and $0 \leq \lambda_{1} \leq \lambda_{2} \leq$ $\lambda_{3} \leq \lambda_{4}$ for the parts of the partition $\lambda$ of $2 n$. They also use the more common combinatorial notation $p_{2}$ rather than $\psi_{2}$.

Theorem 5 of CR98 can be readily restated as follows, by grouping separately the partitions $\lambda$ of $2 a+2 b$ with $\lambda_{1}+\lambda_{3} \geq 2 a$ and those with $\lambda_{1}+\lambda_{3}<2 a$ in the expansion of $\psi_{2}\left(s_{a, b}\right)$ :

- When $\lambda_{1}+\lambda_{3} \geq 2 a, \lambda_{1}+\lambda_{2}$ is even and $\lambda_{1}+\lambda_{2} \leq 2 a$, the Schur function $s_{\lambda}$ has coefficient $(-1)^{\lambda_{2}+\lambda_{3}}$.

- When $\lambda_{1}+\lambda_{3}<2 a, \lambda_{2}+\lambda_{3}$ is even, $2 a \leq \lambda_{2}+\lambda_{3}$ and $2 a \leq \lambda_{1}+\lambda_{4}$, the Schur function $s_{\lambda}$ has coefficient $(-1)^{\lambda_{1}+\bar{\lambda}_{2}}$.

- All other $s_{\lambda}$ have coefficient 0 .

The first of these cases corresponds to the partitions in (ii) and some of (i) in [CR98, Thm. 5], while the second corresponds to the partitions in (iii) and the remaining partitions in (i).

5.2. Reformulation of Carini and Remmel's expansion of $\psi_{2}\left(s_{m_{1}, m_{2}}\right)$. Theorem 5 of CR98 gives rise to an expansion of $\psi_{2}\left(s_{m_{1}, m_{2}}\right), m_{1} \geq m_{2}$, in Schur functions of $x_{1}, \ldots, x_{N}$ which is valid for all $N$.

We can reformulate this further by specifying the support set for the partitions which appear in the expansion in terms of linear inequalities and some parity rules, so that Theorem 4.2, the case where $N=3$, is an immediate corollary.

Using Macdonald's ordering, we take $m_{1}$ in place of $b$ and $m_{2}$ in place of $a$ from CR98 and write $\left(\lambda_{4}, \lambda_{3}, \lambda_{2}, \lambda_{1}\right)=(a, b, c, d)=\lambda$.

Definition 5.1. For $m_{1}, m_{2} \in \mathbb{N}$, let $A\left(m_{1}, m_{2}\right) \subset \mathbb{N}^{4}$ denote the set of tuples $(a, b, c, d)$ that satisfy

- $a+b+c+d=2 m_{1}+2 m_{2}, a \geq b \geq c \geq d \geq 0,2 m_{1} \geq a+d \geq 2 m_{2} \geq c+d$;

- if $b+d \geq 2 m_{2}$, then $c \equiv d \bmod 2$;

- if $b+d \leq 2 m_{2}$, then $a \equiv d \bmod 2$.

Theorem 5.2. Let $m_{1} \geq m_{2} \geq 0$. Then

$$
\psi_{2}\left(s_{m_{1}, m_{2}}\right)=\sum_{(a, b, c, d) \in A\left(m_{1}, m_{2}\right)}(-1)^{b+d} s_{a, b, c, d} .
$$

Theorem 4.2 is an immediate corollary, since Schur functions for partitions with more than 3 rows are 0 in $\mathfrak{s l}_{3}$, and the support set $A\left(m_{1}, m_{2}\right)$ becomes $D\left(m_{1}, m_{2}\right)$ when $d=0$.

We can see readily that Theorem 5.2 follows from Theorem 5 of [CR98] as rearranged above. 
Firstly, for $\lambda \in A\left(m_{1}, m_{2}\right)$ with $b+d \geq 2 m_{2}$ we have $c+d$ even, by the parity rule, and $c+d \leq 2 m_{2}$, while the coefficient of $s_{\lambda}$ is $(-1)^{b+d}=(-1)^{b+c}$. This agrees with the first group of partitions above. The condition $2 m_{1} \geq a+d$ does not impose any extra restriction on this group, since it is equivalent to $b+c \geq 2 m_{2}$.

For $\lambda \in A\left(m_{1}, m_{2}\right)$ with $b+d \leq 2 m_{2}$ we have $a+d$ even, and hence $b+c$ even, by the parity rule. In addition we have $2 m_{2} \leq b+c$ since $2 m_{1} \geq a+d$, and $2 m_{2} \leq a+d$. Again this agrees with the second group of partitions above, and the coefficient of $s_{\lambda}$ is $(-1)^{b+d}=(-1)^{c+d}$ as required there.

5.3. Parametrisation. Theorem 5.2 can be used to give a parametrisation of these two sets of Schur functions with nonzero coefficient, each in terms of 3 integer parameters satisfying some linear inequalities. These in turn give a parametric formula for $\psi_{2}\left(s_{m_{1}, m_{2}}\right)$, with a reduction in the case of $\mathfrak{s l}_{3}$ to the formulae of Theorem 2.4

5.3.1. The first group of Schur functions. Parametrise $\left\{A\left(m_{1}, m_{2}\right): b+d \geq 2 m_{2}\right\}$ by setting $b+d=2 m_{2}+k, k \geq 0$. Write $c=d+2 l, l \geq 0$ to get $c \equiv d \bmod 2$. The condition $c+d \leq 2 m_{2}$ is equivalent to $d+l \leq m_{2}$. This ensures that $c \leq b$. Then $a=2 m_{1}-k-2 l-d$, which satisfies $2 m_{1} \geq a+d$. To ensure that $a \geq b$ we impose the condition $a+d=2 m_{1}-k-2 l \geq b+d=2 m_{2}+k$ to finish with parameters $k, l, d \geq 0, d+l \leq m_{2}, k+l \leq m_{1}-m_{2}$.

The contribution of the partitions $\lambda$ with $b+d \geq 2 m_{2}$ is then

$$
\sum(-1)^{k} s_{\lambda}, \text { where } \lambda=\left(2 m_{1}-k-2 l-d, 2 m_{2}+k-d, 2 l+d, d\right)
$$

and $k, l, d$ are integer parameters with $k, l, d \geq 0, d+l \leq m_{2}, k+l \leq m_{1}-m_{2}$.

5.3.2. The second group of Schur functions. Parametrise $\left\{A\left(m_{1}, m_{2}\right): b+d \leq 2 m_{2}\right\}$ by setting $b+d=2 m_{2}-k, k \geq 0$. Write $a+d=2 m_{1}-2 l, l \geq 0$ to get $a \equiv d \bmod 2$ and $2 m_{1} \geq a+d$. Then $b+c=2 m_{2}+2 l$, so $c \geq d$. The condition $2 m_{2} \leq a+d$ is equivalent to $l \leq m_{1}-m_{2}$. This ensures that $b \leq a$.

Now $b=2 m_{2}-k-d$, so $c=2 l+k+d$, so $c \leq b$ is equivalent to $l+k+d \leq m_{2}$.

The contribution of the partitions $\lambda$ with $b+d \leq 2 m_{2}$ is

$$
\sum(-1)^{k} s_{\lambda}, \text { where } \lambda=\left(2 m_{1}-2 l-d, 2 m_{2}-k-d, 2 l+k+d, d\right)
$$

and $k, l, d$ are integer parameters with $k, l, d \geq 0, l+k+d \leq m_{2}, l \leq m_{1}-m_{2}$.

5.4. Reduction to the case of $\mathfrak{s l}_{3}$. In the special case of $\mathfrak{s l}_{3}$ we have $d=0$, and we get two double sums of 3-row Schur functions, one for partitions with $b \geq 2 m_{2}$, and one for those with $b<2 m_{2}$, to avoid double counting those with $b=2 m_{2}$. Since we are working in $\mathfrak{s l}_{3}$ this can be reduced further to sums over 2-row partitions, since $s_{a, b, c}=s_{a-c, b-c}$.

Explicitly we have from the first group of partitions the sum

$$
\sum(-1)^{k} s_{2 m_{1}-4 l-k, 2 m_{2}-2 l+k}
$$

taken over $k, l \geq 0, l \leq m_{2}, k+l \leq m_{1}-m_{2}$. The second group yields

$$
\sum(-1)^{k} s_{2 m_{1}-4 l-k, 2 m_{2}-2 l-2 k}
$$

taken over $l \geq 0, k>0, k+l \leq m_{2}, l \leq m_{1}-m_{2}$. This gives a second proof of Theorem 2.4. It may be preferable all the same to retain the 3 -row format when estimating the effects of twists in $\mathfrak{s l}_{3}$ as then all the partitions have $2 m_{1}+2 m_{2}$ cells and thus their twist factors depend only on the total content of the partition. 


\section{Sample computations}

In this section we give some sample computations of Theorems 2.1 and 2.4 . Theorem 2.1 implies that:

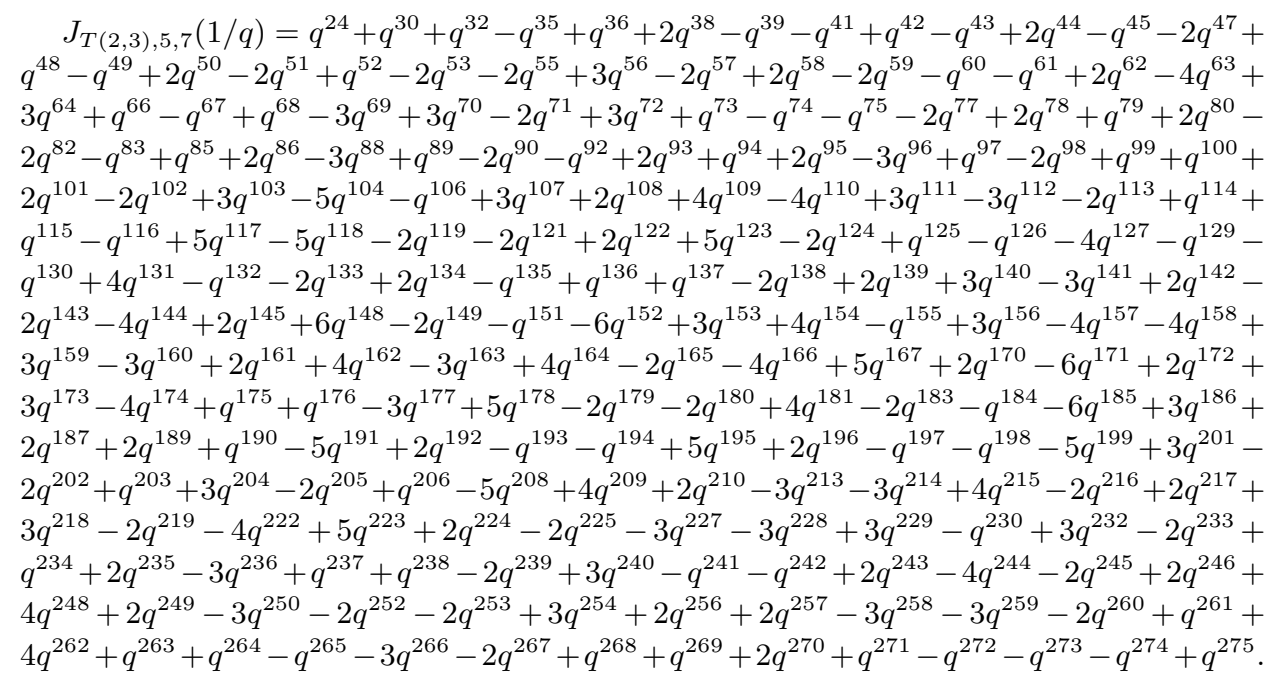

Theorem 2.4 implies that:

$\psi_{2}\left(V_{5,7}\right)=V_{0,4}-V_{0,7}+V_{0,10}-V_{0,13}+V_{0,16}-V_{0,19}-V_{1,2}+V_{2,0}+V_{2,6}-V_{2,9}+V_{2,12}-$ $V_{2,15}+V_{2,18}-V_{3,4}+V_{4,2}+V_{4,8}-V_{4,11}+V_{4,14}-V_{4,17}-V_{5,0}-V_{5,6}+V_{6,4}+V_{6,10}-V_{6,13}+$ $V_{6,16}-V_{7,2}-V_{7,8}+V_{8,0}+V_{8,6}+V_{8,12}-V_{8,15}-V_{9,4}-V_{9,10}+V_{10,2}+V_{10,8}+V_{10,14}-V_{11,0}-$ $V_{11,6}-V_{11,12}+V_{12,4}+V_{12,10}-V_{13,2}-V_{13,8}+V_{14,0}+V_{14,6}-V_{15,4}+V_{16,2}-V_{17,0}$, where $V_{n_{1}, n_{2}}=V_{n_{1} \omega_{1}+n_{2} \omega_{2}}$.

For future checks with other formulas, Theorem 2.1 implies that $J_{2,3,70,70}(1 / q)$ is a polynomial of $q$ with exponents with respect to $q$ in the interval [280,30100] (where the end points are attained), leading and trailing coefficients 1 and coefficients in the interval $[-55196,65594]$, where the coefficient -55196 is attained at precisely $q^{18854}$ and $q^{18925}$ and the coefficient 65594 is attained precisely at $q^{18165}$. In other words, we have

$$
\begin{aligned}
J_{2,3,70,70}(1 / q)= & q^{280}+\cdots+65594 q^{18165}+\cdots-55196 q^{18854}+\cdots-55196 q^{18925} \\
& +\cdots+q^{30100} .
\end{aligned}
$$

Using Theorem 2.1 it is possible to compute the colored Jones polynomials $J_{T(2,3), n_{1}, n_{2}}(q)$ for $n_{1}, n_{2}=0, \ldots, 100$.

\section{ACKNOWLEDGMENTS}

This paper came to maturity following requests for explicit formulas for the colored Jones polynomial of a knot during visits of the first author in the MaxPlanck-Institut für Mathematik in 2009-2010 and during an Oberwolfach workshop in August 2010. The first author wishes to thank R. Lawrence and D. Zagier for their interest, J. Stembridge for enlightening conversations, and the organizers of the Oberwolfach workshop 1033/2010, P. Gunnells, W. Neumann, A. S. Sikora and D. Zagier, for their superb hospitality. 


\section{REFERENCES}

[BN05] Dror Bar-Natan, Knotatlas, 2005, http://katlas.org.

[CGR84] Y. M. Chen, A. M. Garsia, and J. Remmel, Algorithms for plethysm, Combinatorics and algebra (Boulder, Colo., 1983), Contemp. Math., vol. 34, Amer. Math. Soc., Providence, RI, 1984, pp. 109-153. MR777698 (86f:05010)

[CR98] Luisa Carini and J. B. Remmel, Formulas for the expansion of the plethysms $s_{2}\left[s_{(a, b)}\right]$ and $s_{2}\left[s_{\left(n^{k}\right)}\right]$, Discrete Math. 193 (1998), no. 1-3, 147-177, Selected papers in honor of Adriano Garsia (Taormina, 1994). MR1661367 (2000b:05129)

[FH91] William Fulton and Joe Harris, Representation theory, Graduate Texts in Mathematics, vol. 129, Springer-Verlag, New York, 1991, A first course, Readings in Mathematics. MR 1153249 (93a:20069)

[GK10] Stavros Garoufalidis and Christoph Koutschan, The $\mathfrak{s l}_{3}$ Jones polynomial of the trefoil: a case study of q-holonomic sequences, Advances in Applied Math. 47 (4) (2011), 829839. MR 2832380

[GV] Stavros Garoufalidis and Thao Vuong, The degree conjecture for torus knots, preprint, 2010.

[Jan96] Jens Carsten Jantzen, Lectures on quantum groups, Graduate Studies in Mathematics, vol. 6, American Mathematical Society, Providence, RI, 1996. MR1359532 (96m:17029)

[Jon87] V. F. R. Jones, Hecke algebra representations of braid groups and link polynomials, Ann. of Math. (2) 126 (1987), no. 2, 335-388. MR908150 (89c:46092)

[Law03] Ruth Lawrence, The PSU(3) invariant of the Poincaré homology sphere, Proceedings of the Pacific Institute for the Mathematical Sciences Workshop "Invariants of ThreeManifolds" (Calgary, AB, 1999), Topology Appl. 127 (2003), 153-168. MR.1953324 (2003m:57032)

[Mac95] I. G. Macdonald, Symmetric functions and Hall polynomials, second ed., Oxford Mathematical Monographs, The Clarendon Press, Oxford University Press, New York, 1995, with contributions by A. Zelevinsky, Oxford Science Publications. MR 1354144 (96h:05207)

[MM08] H. R. Morton and P. M. G. Manchón, Geometrical relations and plethysms in the Homfly skein of the annulus, J. Lond. Math. Soc. (2) 78 (2008), no. 2, 305-328. MR2439627 (2009h:57021)

[Mor95] H. R. Morton, The coloured Jones function and Alexander polynomial for torus knots, Math. Proc. Cambridge Philos. Soc. 117 (1995), no. 1, 129-135. MR.1297899 (95h:57008)

[RJ93] Marc Rosso and Vaughan Jones, On the invariants of torus knots derived from quantum groups, J. Knot Theory Ramifications 2 (1993), no. 1, 97-112. MR.1209320 (94a:57019)

[Tur88] V. G. Turaev, The Yang-Baxter equation and invariants of links, Invent. Math. 92 (1988), no. 3, 527-553. MR939474 (89e:57003)

[Tur94] _ Quantum invariants of knots and 3-manifolds, de Gruyter Studies in Mathematics, vol. 18, Walter de Gruyter \& Co., Berlin, 1994. MR1292673 (95k:57014) 0160

School of Mathematics, Georgia Institute of Technology, Atlanta, Georgia 30332-

E-mail address: stavros@math.gatech.edu

$U R L:$ http://www.math.gatech.edu/ stavros

Department of Mathematics, University of Liverpool, Liverpool L69 3BX, England

E-mail address: morton@liverpool.ac.uk

URL: http://www.liv.ac.uk/ su14

School of Mathematics, Georgia Institute of Technology, Atlanta, Georgia 303320160

E-mail address: tvuong@math.gatech.edu

$U R L:$ http://www.math.gatech.edu/ tvuong 